SEVERE IMMUNE THROMBOCYTOPENIA IN A PATIENT WITH HIV-HCV COINFECTION

\title{
General Medicine
}

Dr. R. Murugaraj* (PG) Professor.*Corresponding Author

\section{Dr. V. Padma \\ Dr. Rameez Raja (AP)}

\section{KEYWORDS}

\section{Thrombocytopenia}

Thrombocytopenia in a patient with HCV - HIV co-infection can hav e a multitude of causes. Thrombocytopenia is seen in $5-30 \%$ of HIV patients. 1 The most common cause of thrombocytopenia in HIV patients is HIV-associated thrombocytopenia. The aetiology for HIV associated thrombocytopenia is complex and is due to both immune mediated peripheral destruction of platelets as well is reduced platelet production from the progenitor cells. However secondary causes of thrombocytopenia needs to be ruled out in all patients with HIV infection. Common secondary causes for thrombocytopenia include infections (viral, bacterial, fungal and parasitic), malignancies, drugs, hypersplenism and other causes of cirrhosis. In our patient no secondary cause could be found except for co- infection with Hepatitis C virus.

Various studies have shown that $10-36 \%$ of chronic ITP patients have und er l yi ng $\mathrm{c} h$ ro $\mathrm{n}$ ic $\mathrm{H}$ e pa titi s $\mathrm{C}$ infection.1,3-6 Another study has shown that $\mathrm{HCV}$ infection is associated with an elevated risk of developing ITP.7 Various mechanisms has been postulated for HCV associated thrombocytopenia. Immune mediated peripheral destruction and hypersplenism secondary to portal hypertension are major causes. 7

Our patient thus had HIV-HCV co- infection. All opportunistic infections and malignancies were ruled out. Ultrasound abdomen showed no evidence of cirrhosis or splenomegaly. A diagnosis of immune mediated thrombocytopenia due to HIV/HCV co- infection was thus made. Considering the severe thrombocytopenia with bleeding manifestation the patient was given Intravenous Immunoglobulin $1 \mathrm{~g} / \mathrm{kg}$ single dose. Steroids were initially not given considering the flare up of underlying occult opportunistic infections and possible progressive liver damage due to Hepatitis C. He was also simultaneously started on ART and antiviral therapy for HCV.

Within 48 hours of initiation of IVIG, his platelet count improved reaching a maximum of $60,000 / \mathrm{mm} 3$ on the fifth day. Thereafter the platelet count started falling again and reached a level of $6000 / \mathrm{mm} 3$. It was decided to give a second dose of IVIG along with the addition of a second agent for long term management of ITP. Thrombopoietin receptor agonist, eltrombopag was considered in this patient. Eltrombopag was found to increases platelet numbers in thrombocytopenic patients with $\mathrm{HCV}$ and advanced fib rosis and cirrhosis in randomised trials.8,9 The major side effect concern is the risk of thromboembolic event like portal vein thrombosis.9 Data regarding the use of the thrombopoetin receptor agonists, eltrombopag and romiplostim in HIV associated thrombocytopenia is limited. In five patients of refractory ITP associated with HIV, thrombopoetin receptor agonists improved platelet count in all of them. Two of them however succumbed to thromboembolic complications. 10 We should therefore be careful when using these agents in patients with thromboembolic risk factors including hypertension, smoking, coronary artery disease, and obesity. Our patient did not have any thromboembolic risk factors and thus was a potential candidate for eltrombopag. However our patient could not afford the drug.

Second option considered in the patient was oral glucocorticoids. Prednisolone has been found to be effective in increasing platelet count in patients with HIV ass ociated thrombocytopenia. However only a minority of patients will have sustained increase in platelets after cessation of steroids. Short term steroid use in these patients were not associated with any increased risk of infections. HCV related immune thrombocytopenia has also been shown to be responsive to steroids. However there is a risk of progression of liver disease in HCV patients who are treated with steroids.

Considering all these factors including his financial status, he was started on oral prednisolone $(1 \mathrm{mg} / \mathrm{kg})$ with frequent monitoring of liver function. With second dose of IVIG his platelet count increased $2,40,000 / \mathrm{mm} 3$. It was decided to taper the steroids once the platelet count are stable, expecting that the underlying primary pathology will be controlled by the antiviral drugs for HIV and HCV. On follow-up of the patient his platelet count was maintained between 80,000 to $90,000 / \mathrm{mm} 3$ and prednisolone were gradually tapered and stopped over a period of three months.

\section{REFERENCES}

1. Stasi R, Willis F, Shannon MS, Gordon-Smith EC. Infectious causes of chronic immune thrombocytopenia. Hematol Oncol Clin North Am 2009; 23:1275-97. 